\title{
Artificial Digitality (A Musical Journey to the Future)
}

\author{
Kuldeep Gohel* \\ Digital Educator at AMHN, NYC and UX Designer at Fidelity Investment, NJ. Indian. \\ * Corresponding author. Tel.: +1 (732)-979-3089; email: gohek510@newschool.edu \\ Manuscript submitted January 15, 2019; accepted March 6, 2018. \\ doi: $10.17706 /$ jcp.14.3.232-239
}

\begin{abstract}
This document discusses the technical process and artistic intent of a concept music album co-authored by a human and Artificial Intelligence. The project finishes with an album of several compositions. The album begins with a composition that is generated by the author alone, a self-taught musician [1] and a technologist. The compositions that follow are co-authored by an open source neural network and the author. The neural network is trained by the author [2], who has turned his compositions into mathematical data which can be fed to the neural network. The album ends with a composition that is completely generated by the trained neural network. The goal of the project is to express the rise of Artificial Intelligence in a musical way and speculate on the future of Artificial Intelligence. The author uses music, mathematics, and the emerging machine learning field to create a musical story. It aims to question about the future where automation takes over the human labor in various fields including creative areas like music production and art making.
\end{abstract}

Key words: Artificial intelligence, conceptual computer art, music, story telling.

\section{Context}

The project is driven by two forces: the author's love for music composition and artificial intelligence. The author is a self-taught musician who has indulged himself in the process of music composition for a long time. For the past five years, the author has been constantly working on creating music and has recently ventured into using $\mathrm{AI}$ as a tool for creating music.

The author in these five years of music learning has been involved in various other studies related to emerging technologies and several art and design projects. In this time, he heard about Artificial Intelligence and fell in love with the idea that a machine can replicate him and help him to produce music. He considers machine learning as a tool to replicate his brain and generate a body in the computer that he can share his soul with.

This thinking has led him to work on researching about Artificial Intelligence [3] and machine learning that he supposes will be taking over the world very soon. The author's constant effort to achieve a system that can replicate his style of music production has finally led him to a neural network and a mathematical system that clones his musical passion.

The author aims to generate an album where machine learning is used to make music and the compositions are used as a medium to express the rise of Artificial Intelligence. Along with this he aims to speculate on the future of Artificial Intelligence and figure out the possible ways Artificial Intelligence will assist humans in the future. 


\section{Idea}

\subsection{Conception}

The very first idea/subject that forms the story line for this project was to express the rise of Artificial Intelligence through Concept Music Album. The aim of this project was to build a musical narrative that speaks of the advent of AI and the role of human labour in this context.

"There is no reason and no way that a human mind can keep up with an artificial intelligence machine by 2035."

Gray Scott

The idea was broken down as follows:

Story/Subject/Theme: The rise of Artificial Intelligence and speculate its future in our planet.

Medium: Concept Music Album.

The aim was to narrate how the world has been changing with the advent of Artificial Intelligence through a concept music album. Each composition in the album expresses the world of that period of time. This period of time was decided on the basis of the birth period of Artificial Intelligence. It was John McCarthy, in 1956, who coined the term "Artificial Intelligence" as the topic of the Dartmouth Conference, the first conference devoted to the subject; Artificial Intelligence.

Apart from the working history of Artificial Intelligence which is dated by to 1956, the concept of machines carrying human intelligence and the roots of AI can be found in various mythologies around the world. For example, the intelligent bronze man Talos, made by Hephaestus. Humans have long sought to create human-like artefacts as demonstrated by the works of Archytas, Hero and Daedalus.

\subsection{Simplification}

The research about the birth period of Artificial Intelligent and its rise led to simplification of the album:

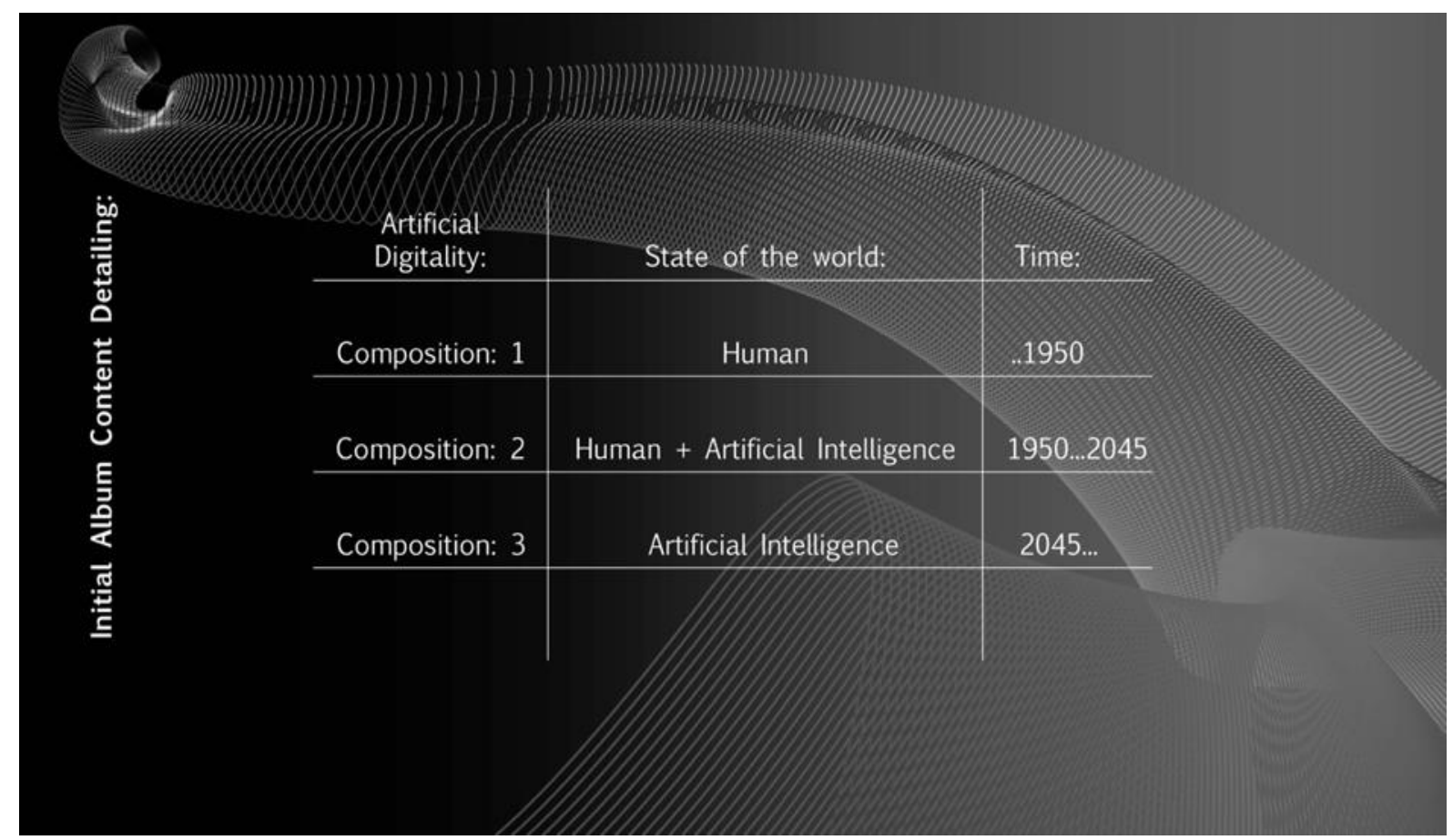

Fig. 1. Initial album content detailing. 


\subsection{Concept Refinement}

The previous breakdown of the album had a correct time period and the story narration that it was intended to narrate through the music pieces. The composition one, was describing the world where Humans were ruling, the composition second was describing the current world where Humans and Artificial Intelligence: both are playing their role for the smooth functioning of the planet Earth. While the thirst composition is describing the singularity state of rising. So the album starts with the description of the world where the Humans are the ruling party and ends with the world where the Artificial Intelligence has taken over the world and all the tasks that requires human intelligence are performed by intelligent mechanical agents.

The fine reviewing of the concept led to a minor but an impactful change in the album composition process; if the album is describing how the world will be taken over by the Artificial Intelligence and the human tasks will be done by machines then the act of music composing here, should also be taken over by the Artificial Intelligence. The medium should be turning out as the message and so the composer also along with the composition progression will go from human to Artificial Intelligence.

This led to a refined breakdown of the concept and the album; as shown in the image in the previous page. This even led to the addition of the new domain; apart from the two obvious ones: Music and Artificial Intelligence. As Machine Learning was to be used as a tool to compose music, it become the specific domain under the Artificial Intelligence which will be studied in terms of it technical aspects like data sets, algorithms and mathematics.

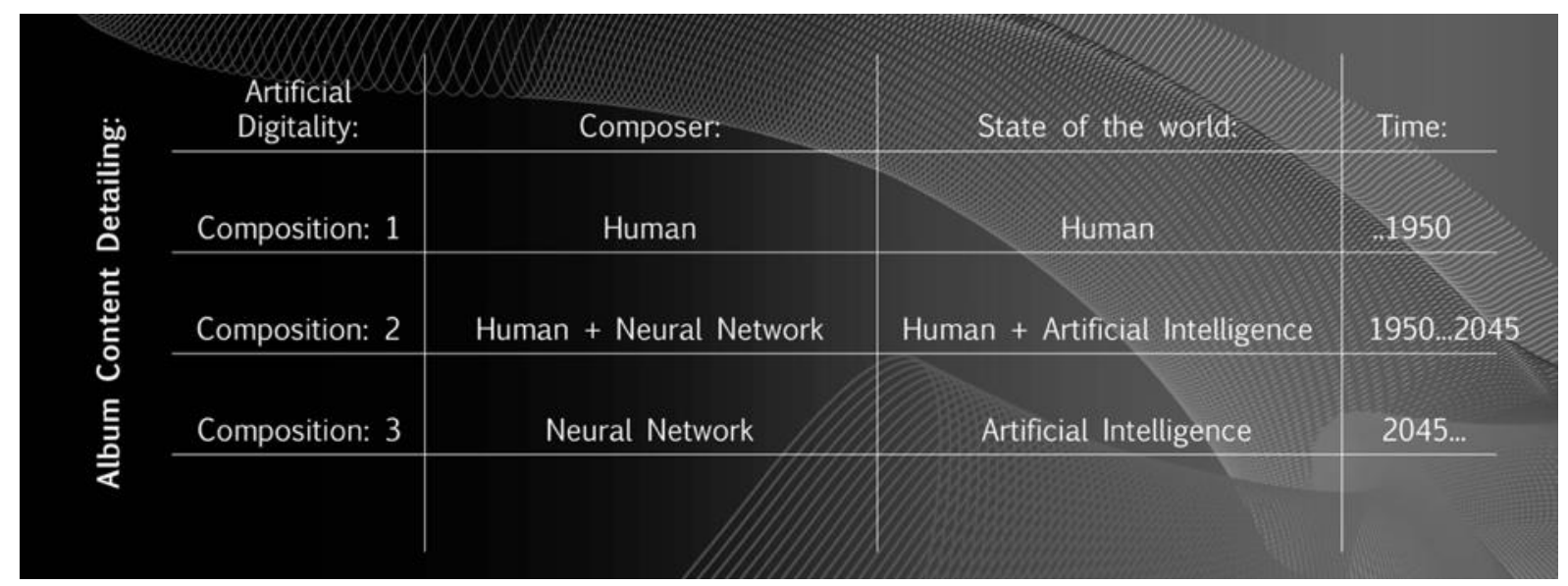

Fig. 2. Album content detailing.

\section{Process}

The process involved an analytical approach [4] to the art of music making. The author analyzed the process of making music, then gathered and converted the process into data; number and sequences, which can be used to generate a system that will clone his music making.

Author started by dividing the music album in three compositions and the content of each composition draws inspiration from the story of the development of Artificial Intelligence [5] to date. So three compositions, with the subject: 'World before Artificial Intelligence', 'Current World' and 'Future (Singularity)' were to be generated. The three compositions were generated by: only author, author and his neural network and only neural network respectively, just like the changing face of the world with the advent of Artificial Intelligence. To eliminate the error, each composition was limited to two octaves of a specific scale, preselected to give out certain emotions associated with the subject of narration. So the composition 'World before Artificial Intelligence' was created in C Natural Minor (90 beats per minutes), 
while keeping Sentimental and Tragic as the emotion of the narration. For 'Current World' and 'Future (Singularity)', C\# Melodic Minor (111 beats per minutes) and G\# Hungarian Gypsy (128 beats per minutes) scales were used and they were to trigger Intellectual and Futuristic emotions respectively. Each of the composition were to be generated from the total of 15 keys offered in two octaves of their scale.

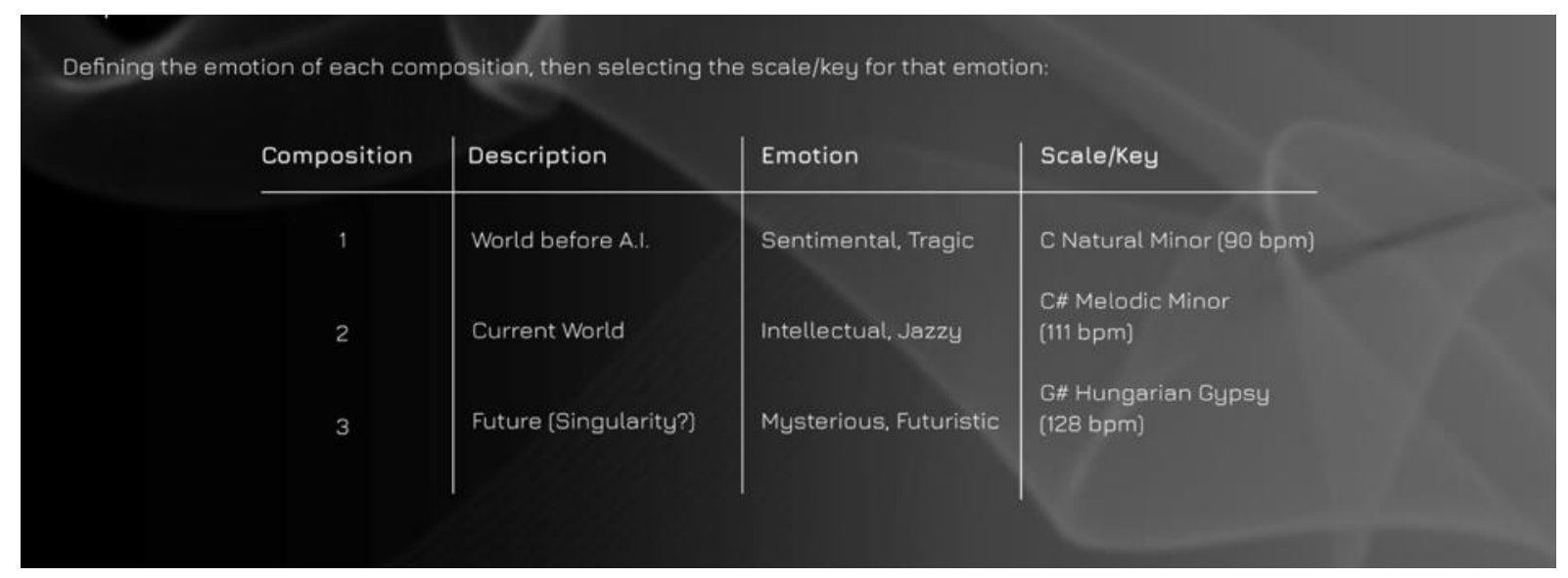

Fig. 3. Scale, emotion and compositions.

Author than created the first composition of 60 seconds, that involved chords and melody aiming to narrate the story of our world before the birth of Artificial Intelligence. Chords (limiting to 3 notes played at once) had one variable which was the sequence of notes on the piano while melody had two variables; time and note sequence. The key's in the selected two octaves of $C$ Natural Minor piano scale were numbered from 1-15 and these numbers were used to get the sequence from the author's first composition [6].

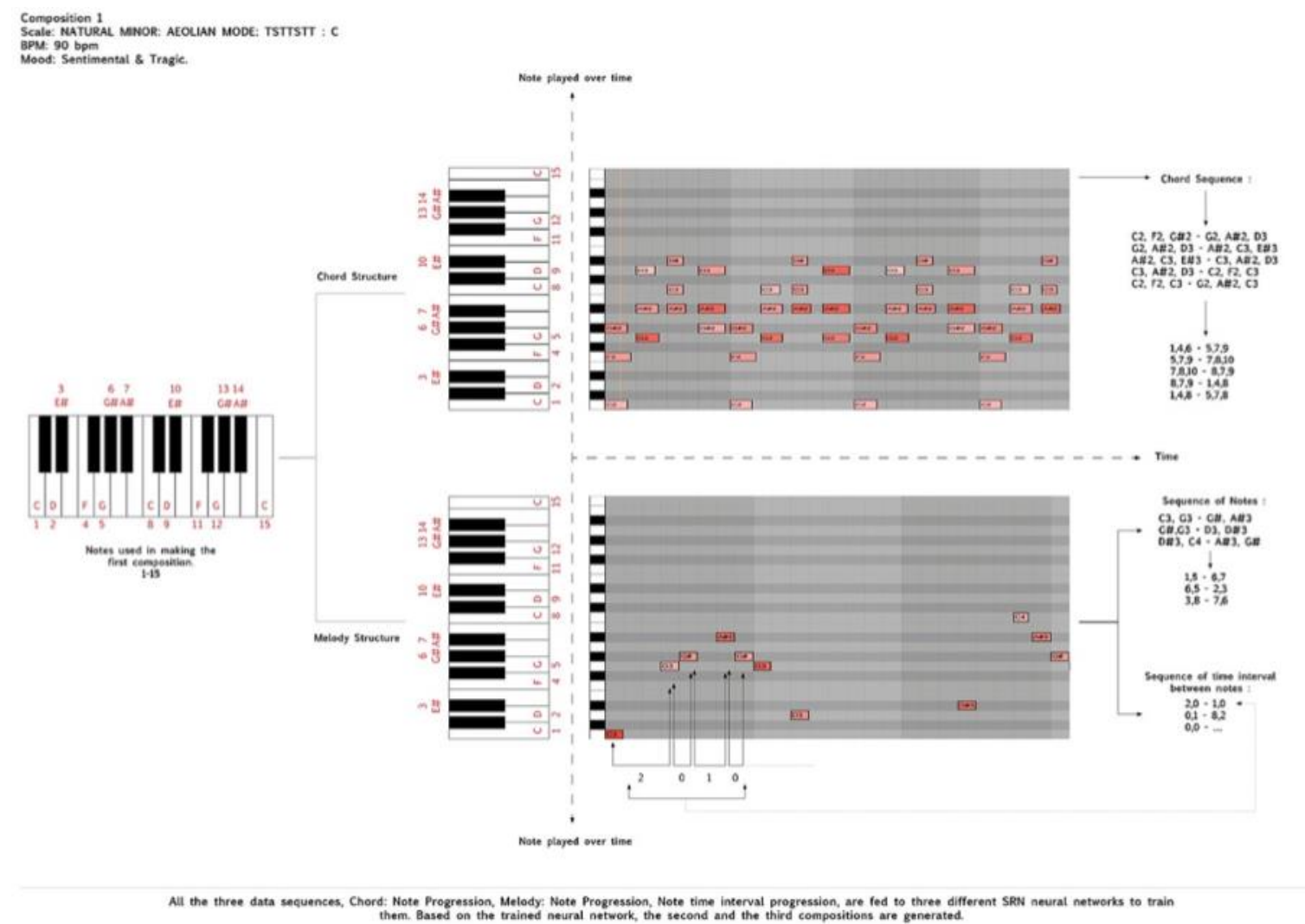

Fig. 4. Composition:1 process. 
Three kinds of sequences were extracted; the chord notes, the time and notes in melody.

Chord Data Extraction:

Chord Progression: Value Range: 1 - 15

$1,4,6-5,7,9$

$5,7,9-7,8,10$

$7,8,10-8,7,9$

$1,4,8-5,7,8 \ldots$

Melody Data Extraction:

1) Melody Note Progression: Value Range: 1 - 15

$1,5-6,7$

$6,5-2,3$

$3,8-7,6$

$12,13-13,8 \ldots \ldots$

2) Melody: Time between two notes progression: Value Range: 0 - 8 (Unit: Bars)

$2,0-1,0$

$1,8-2,0$

$0,1-2,0$

$8,2-0,0 \ldots$

The value of these sequence was normalized between 0 - 1 using (1).

Normalizing all the values between 1-15 and 0 - 8, for decreasing the error margin.

Normalizing Range: 0 - $1(a=0, b=1)$

$a+(x-A)(b-a) /(B-A)=$ Normalized Value.

$x=$ the number (between $A-B)$ whose normalized value is needed.

Chord and Melody Note Range: (1-15). $(A=1, B=15)$

1 - 0,2 - 0.0714 - Example: $0+(2-1)(1-0) /(15-1)=1 / 14=0.0714,3-0.1428,4-0.2142,5-0.2857$

$6-0.3571$ - Example: $0+(6-1)(1-0) /(15-1)=5 / 14=0.3571,7-0.4285,8-0.5,9-0.5714,10-0.6428,11-$ 0.7142 ,

$12-0.7857,13-0.8571,14-0.9285,15-1$

Melody Time Range: $(0-8) \cdot(A=0, B=8)$

0 - 0 - Example: $0+(0-0)(1-0) /(8-0)=0 / 8=0$

1 - 0.125 - Example: $0+(1-0)(1-0) /(8-0)=1 / 8=0.125,2-0.25,3-0.375,4-0.5$,

5 - 0.625 - - Example: $0+(5-0)(1-0) /(8-0)=5 / 8=0.625,6-0.75,7-0.875,8-1.0$

These sequences (with the normalized value) where then used to make data sets (input and target data) to feed to the Simple Recurrent Network (SRN) generated in an open source software; Simbrain as shown in Fig. 5. Three neural networks were trained to give three different sequences; for chord notes, melody notes and melody time.

The trained system was finally used to make second and third compositions. So the first one was composed by the author, the second one by the Artificial Intelligence and the author, and the album concludes with a music piece completely generated by the trained neural network. 


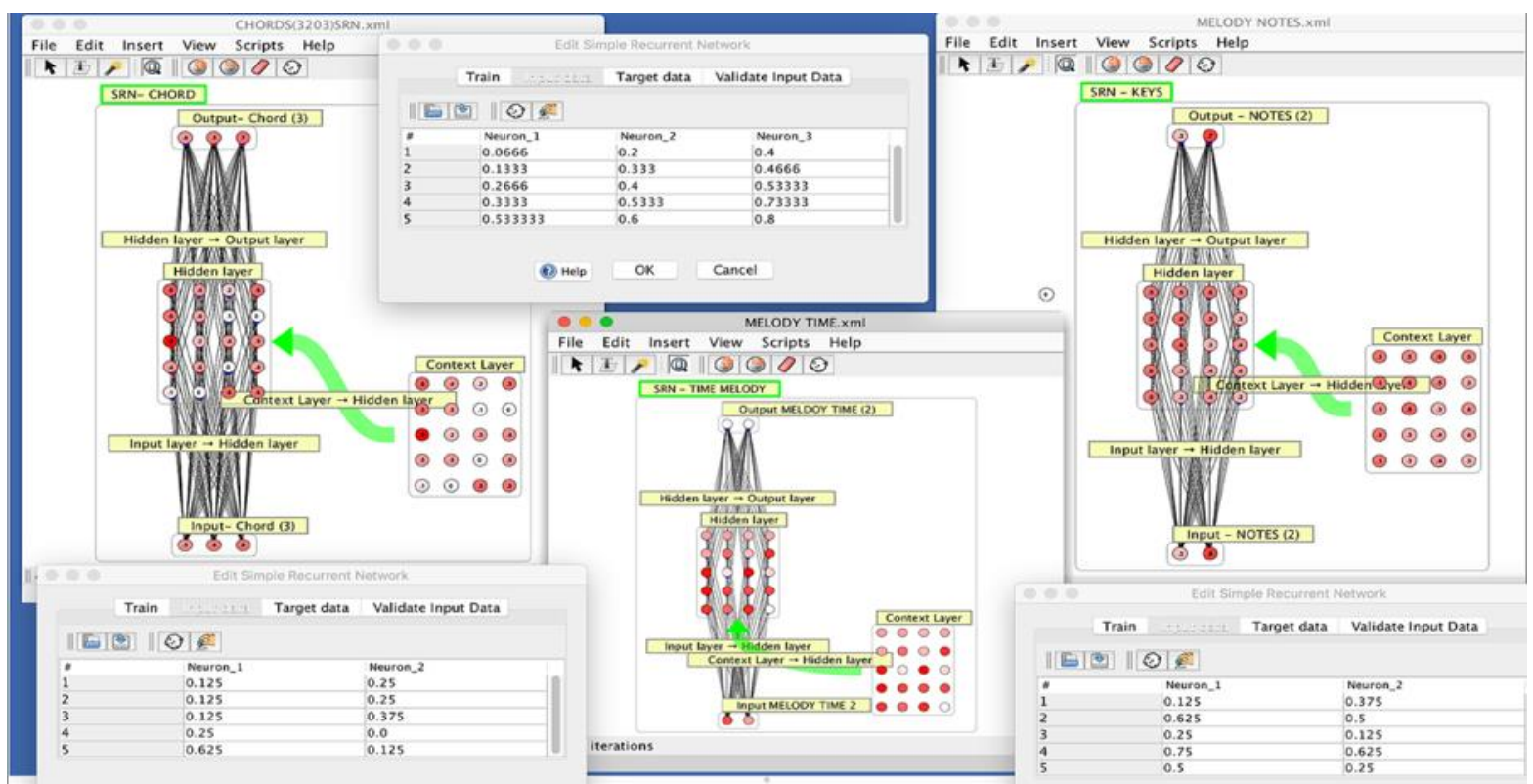

Fig. 5. Neural netwroks.

\subsection{Second Composition}

Composer: Human and Artificial Intelligence.

Changes: Scale and Tempo: Altered Scale: TSTTTTS: C-sharp/D-flat melodic minor. (111 beats per minute). Chord: Feeding value of first chord played by human in the neural network to get the suggestion about the next chord. Third chord decided by the human, fourth by the Neural Network and so on.

Melody: Note Progression: Feeding value of first and second note played by human in the neural network to suggest the third and the fourth note. Fifth and sixth note decided by the human, seventh and the eight by the Neural Network and so on.

Time between the note's progression: Simultaneously with the notes progression, the third neural network is used to get the time intervals between the notes. So the time interval between the 1-2, 2-3 note is decided by the human and fed to the neural network to get value of the time interval between 3-4, 4-5. And so on.

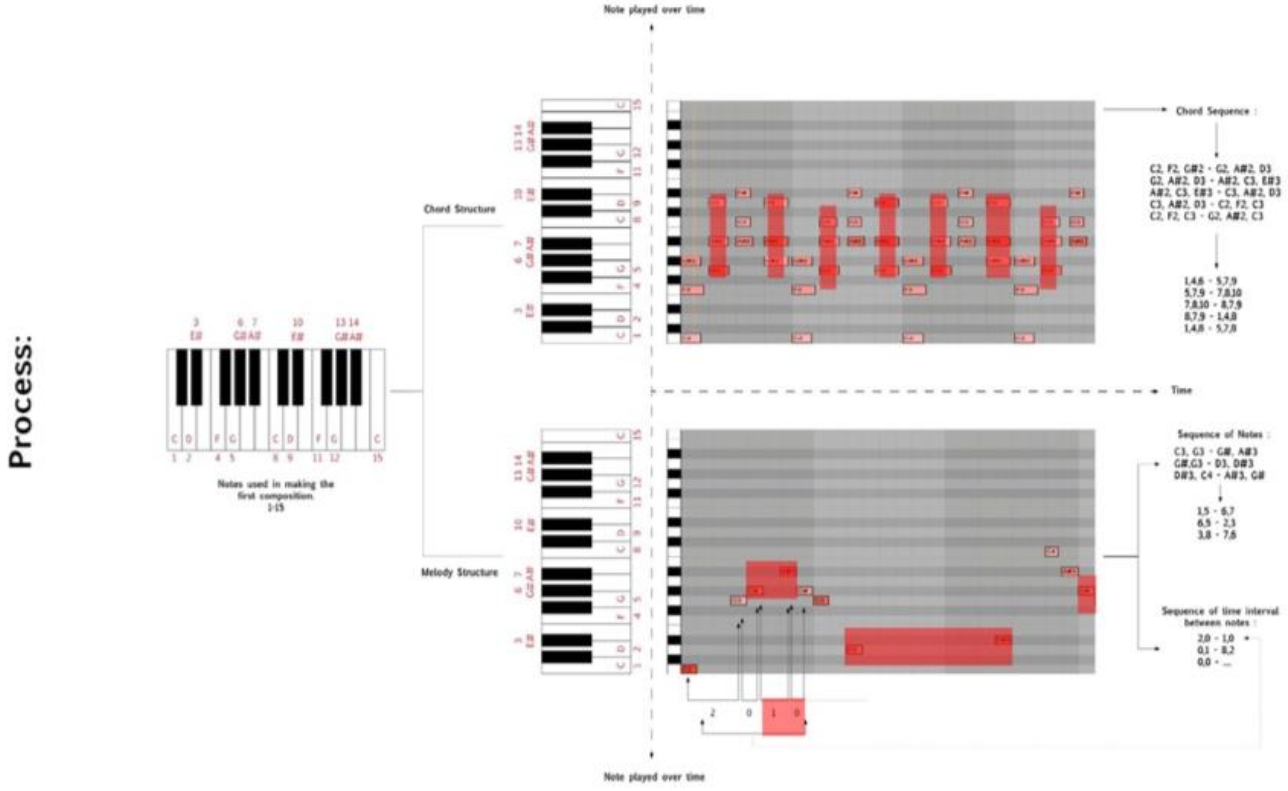

Fig. 6. Composition 2 process (red marked content is generated by artificial intelligence). 


\subsection{Third Composition}

Composer: Only Artificial Intelligence (Neural Network).

Changes: Scale and Tempo: Hungarian Gypsy Scale: TSTSTTS: G \#. (128 beats per minute).

Chord: Feeding value of first chord played by neural network in the neural network to suggest the next chord. Then feeding the value of the second chord by the neural network to get the value of the third and so on. Feeding the value of neural network to itself, to continue getting the successive data.

Melody: Note Progression: Feeding value of first two notes played by neural network in the neural network to suggest the next two notes. Then feeding the value of the third and fourth by the neural network to get the value of the fifth and the sixth note. Feeding the value of neural network to itself, to continue getting the successive data.

Time between the note's progression: Simultaneously with the notes progression, the third neural network is used to get the time intervals between the notes. So the time interval between the 1-2, 2-3 note is decided by the neural network and fed to the neural network to get value of the time interval between 3-4, 4-5. And so on.

\section{Final Production}

\subsection{Graphics - Album Cover}

The visual language [7] is derived from the project concept; how computers started their journey from ' 0 ' and now in the future, they will capable enough to perform creative tasks like composing music; shown in Fig.7 [8].

\subsection{Composition Nomenclature}

Name of each composition derives itself from the different period of time, that the composition is describing:

1)The world before centuries, where necessity began to fuel inventions.

2)The current modern world, where we are exploring the unknown driven by curiosity. But as it has positive and negative both impacts, it becomes a funny trouble.

3)How will you define the future world, where machines and algorithms have taken over humans? Or will we even be in a position to define anything?

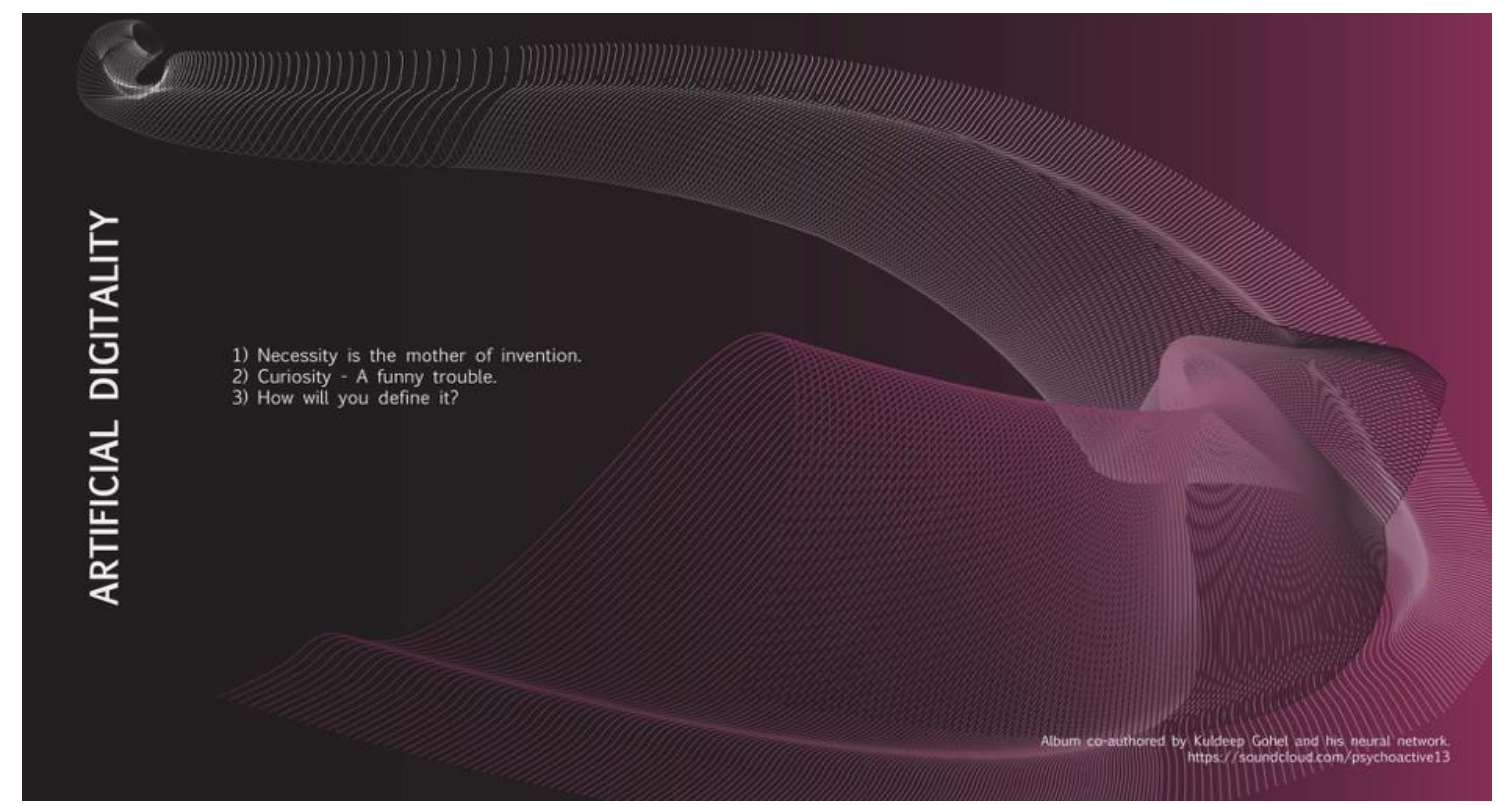

Fig. 7. Album cover. 


\section{Full Composition}

https://soundcloud.com/psychoactive13/sets/artificial-digitality

\section{Conclusion}

Thus, converting a musical narrative into a mathematical structure, allows us the use of novel tools to build unpredictable narratives. Music is a language that can be objectively described and used to create narratives. This objective description allows us to use it to train Artificial Intelligence, that can become future collaborators with creatives to create stories in varying mediums, music being one such medium as demonstrated in this paper.

\section{References}

[1] Schoenberg, A., Strang, G., \& Stein, L. (1999). Fundamentals of Musical Composition. London: Faber and Faber.

[2] Slavio, J. Machine Learning for Beginners.

[3] Alpaydin, E. (2016). Machine Learning: The New AI. Cambridge, MA: The MIT Press.

[4] Burrows, T. (2002). Total Keyboard. New York: Friedman/Fairfax.

[5] Russell, S. J., \& Norvig, P. (2016). Artificial Intelligence: A Modern Approach. Boston: Pearson.

[6] Perricone, J. (2007). Melody in Songwriting: Tools and Techniques for Writing Hit Songs. Boston, MA: Berklee Press.

[7] Powell, A. (2012). Classic Album Covers of the 1970s. London: Collins \& Brown.

[8] Thorgerson, S. (1990). Classic Album Covers of the 60s. Zürich: Olms.

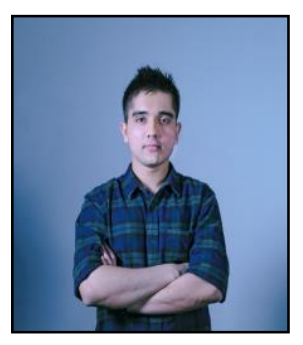

Kuldeep Gohel is a self-learnt musician, creative technologist and a UX Desinger; born in Rajkot, Gujrat India on 13 November, 1992. He graduated in 2018 from Parsons School of Design, NYC where he studied design and technology (MFA). Before studying at Parsons, he did his bachelors from National Institute of Design, Ahmedabad, India in exhibition design, along with a semester exchange at Royal Melbourne Institute of Technology, Melbourne, Australia; where he got major exposure to the power and various faces of weaving art, design and technology together.

He has done art and design shows in Europe, Australia and USA since 2010. And along with this, he has been an active educator. His journey as an educator began from HS OWL, Germany in 2015 and is currently working as a digital educator at AMNH, NYC. Along with being a digital educator, currenlty he works at Fidelity Investment as a UX Desinger in New Jersey.

He has received several awards, the list includes, NYC media Labs 2018 award for data science, MIT media Labs Hackathon award, 2018 and Google cloud API special award as a part of winning Hackathon at MIT media Labs, Boston, USA in 2018. 